DOI: 10.5007/2175-7941.2012v29n3p1121

\title{
ABORDAGEM EXPERIMENTAL DA FORÇA DE ATRITO EM AULAS DE FÍSICA DO ENSINO MÉ- $\mathrm{DIO}^{+*}$
}

Marco Aurélio Alvarenga Monteiro

Isabel Cristina de Castro Monteiro

Alberto Gaspar

Departamento de Física e Química

Faculdade de Engenharia de Guaratinguetá

UNESP

Guaratinguetá - SP

\section{Resumo}

Neste trabalho, propomos e descrevemos a realização de uma atividade experimental para abordar o conceito de atrito em aulas práticas de Física do Ensino Médio. Utilizamos um equipamento de simples construção e de baixo custo que possibilita a determinação do coeficiente de atrito estático entre duas superfícies por meio de três procedimentos distintos. Os resultados obtidos foram coerentes entre si, com pequeno desvio percentual, o que confere confiabilidade à atividade. A atividade também permite uma maior contextualização de conceitos que normalmente são abordados apenas de forma teórica, exigindo um nível maior de abstração dos alunos, o que pode estimular as discussões e uma maior interação entre professor e alunos.

Palavras-chave: Ensino de Física. Força de atrito. Atividade Experimental.

\footnotetext{
Friction force experimental approach in High School Physics classes

* Recebido: julho de 2011.

Aceito: agosto de 2012.
}

Cad. Bras. Ens. Fís., v. 29, n. 3: p. 1121-1136, dez. 2012. 


\begin{abstract}
In this paper we propose and describe the performance of an experimental activity to address the concept of friction in High School Physics practical classes. We use a low-cost and simple construction device that enables the determination of the coefficient of static friction between two materials through three different procedures. The results were coherent, with small percentage deviation, which gives reliability to the activity and can stimulate discussions in class. The activity also allows greater contextualization of concepts that are usually discussed only theoretically, requiring a higher abstraction level of the students. This can stimulate discussions and greater interaction between teacher and students.
\end{abstract}

Keywords: Physics teaching. Friction force. Experimental activity.

\title{
I. Introdução
}

A apresentação em sala de aula de conceitos relacionados ao atrito é, normalmente, limitada à apresentação expositiva de leis e equações empíricas que, em geral, não estimulam a discussão mais detalhada do fenômeno.

Assim, alguns trabalhos têm proposto atividades experimentais simples que possam envolver os alunos de tal forma a motivá-los a se debruçarem-se sobre o assunto em estudos.

Nessa direção, trabalhos como os de Matuo \& Marinelli (2007) e Mossmann et al. (2002), apresentam propostas experimentais para uma abordagem mais efetiva sobre o fenômeno do atrito.

Em convergência com essas propostas, neste trabalho, apresentamos uma atividade baseada em um equipamento de fácil construção e baixo custo que possibilita a determinação do coeficiente de atrito estático por três procedimentos distintos com bons resultados, o qual pode oferecer oportunidades de discussões interes- 
santes acerca do fenômeno do atrito ${ }^{1}$, tanto do ponto de vista qualitativo quanto quantitativo.

Os estudos sobre o atrito datam do final do século $\mathrm{XV}$, com trabalhos realizados por Leonardo da Vinci (veja Fig. 1a). Contudo, as leis empíricas sobre o atrito, válidas ainda hoje, foram formuladas em 1699, por Guillaume Amontons, e complementadas por Charles A. de Coulomb, em 1785.

Coulomb estabeleceu uma clara distinção entre o atrito estático e o atrito cinético que ocorrem quando não há ou há movimento relativo entre as superfícies em contato: a força de atrito cinético é sempre menor do que o a força de atrito estático e, uma vez iniciado o movimento entre duas superfícies em contato, a força de atrito cinético permaneceria constante. Sugeriu, ainda, que o atrito entre superfícies metálicas secas seria devido a encaixes microscópicos entre as superfícies (Fig. 1b), ideia que perdurou até meados do século passado (GASPAR, 2000).

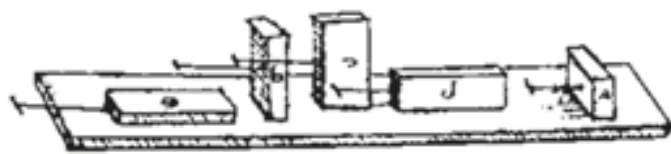

(a)

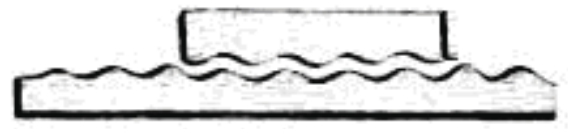

(b)

Fig. 1a-Diagrama original de Leonardo da Vinci que ilustra um de seus experimentos sobre o atrito: a força necessária para deslocar o bloco (representada pelas hastes que puxam os blocos) não depende da área sobre a qual o bloco se apoia (PERSSON, 2000); 1 - Modelo sugerido por Coulomb para a origem do atrito entre duas superfícies metálicas secas, apresentado no livro Théorie de Machine Simples (COULOMB, 1809).

Em 1929, o físico inglês G. A. Tomlinson (1929) propôs a primeira hipótese alternativa para a origem do atrito. De modo muito simplificado, segundo Tomlinson, enquanto uma superfície desliza sobre outra, os átomos que compõem

\footnotetext{
${ }^{1}$ Vamos restringir nossa abordagem ao atrito de arrastamento entre duas superfícies planas e secas; atrito entre superfícies lubrificadas ou em rolamento além de exigirem uma abordagem mais complexa não fazem parte dos currículos de física do ensino médio.
} 
essas estruturas interagem e vibram. É essa vibração que dá origem ao atrito. No entanto, certamente por impossibilidade de verificação experimental, essa hipótese teve pouca repercussão e aceitação.

Em 1950, Bowden e Tabor (1950) verificaram que a área de contato efetivo entre duas superfícies é muito menor do que a área aparente, o que tornou insustentável a hipótese coulombiana dos encaixes.

Segundo Gaspar (opus cit.), essa descoberta levou esses pesquisadores a formularem uma nova hipótese: devido à proximidade entre as moléculas dos materiais em contato, nas pequenas regiões em que ele efetivamente ocorre, surgem forças eletromagnéticas de adesão que "soldam" microparcelas das superfícies que, de fato, estão em contato. Assim, quando uma superfície é arrastada sobre a outra, ocorrem sucessivas "soldas", rupturas e deslizamento, em um processo conhecido por stick-slip (gruda-escorrega), que dá origem à força de atrito.

Com a invenção dos microscópios de força atômica e de tunelamento, e de microbalanças que permitem medir forças de interação extremamente pequenas entre átomos das superfícies, esse modelo para o atrito pôde ser estudado experimentalmente. As pesquisas com esses instrumentos têm proporcionado grande avanço no conhecimento do atrito e têm mostrado que, apesar de a hipótese do stick-slip ser verdadeira, não se pode prescindir da hipótese anterior de Tomlinson, sobretudo quando não há desgaste entre as superfícies.

No entanto, embora a natureza do atrito, ainda hoje seja mal compreendida, as leis empíricas estabelecidas por Amontons e Coulomb continuam sendo aceitas dentro dos limites de validade dos ensaios experimentais em que são aplicadas. Assim, para a atividade experimental aqui proposta, de acordo com uma dessas leis, sendo $N$ o módulo da forma normal às superfícies em contato, $f_{a e}$, o módulo da força de atrito estático entre essas superfícies, pode ser expresso pela inequação:

$$
f_{a e} \leq N \mu_{e}
$$

em que $\mu$ e é o coeficiente de atrito estático que depende, entre outros fatores, da natureza das superfícies em contato.

Considerando o módulo da força de atrito estático máximo, $f_{a e(m a ́ x)}$, essa lei pode ser escrita pela equação:

$$
f_{a e(\operatorname{má} x)}=N \mu_{e}
$$


Quando ocorre o movimento, surge a força de atrito cinético, cujo módulo $f_{a c}$, suposto constante (o que é válido para baixas velocidades), é dado pela equação:

$$
f_{a c}=N \mu_{c},
$$

em que $\mu_{c}$ é o coeficiente de atrito cinético, que depende do material das superfícies em contato, e $\mathrm{N}$ é o módulo da força normal entre as superfícies em contato, como está indicado na Fig. 2.

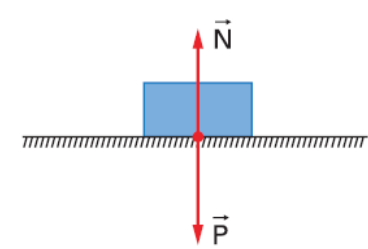

(a)

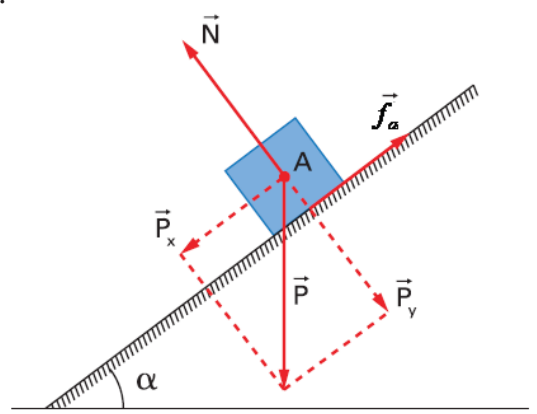

(b)

Fig. 2 - (a) Em módulo, $N=P$; (b) $N=P_{y}$.

Em qualquer um desses casos, a força normal é sempre perpendicular às forças de atrito entre as superfícies em contato, por isso não é possível exprimir essas relações na forma vetorial. Em a, não está representada nenhuma força exercida sobre o bloco no sentido de deslocá-lo sobre o plano, por isso não foi representada a força de atrito entre as superfícies; em b, a força de atrito está representada por causa da ação do componente do peso do bloco, $P_{x}$, que sempre tende a deslocá-lo para baixo. Não há, no entanto, indicação da natureza da força de atrito (estático ou cinético) porque, nessa situação, o bloco pode ou não estar em movimento, o que ocorre quando o ângulo de inclinação $\alpha$ supera o ângulo crítico $\theta_{c}$, dado pela expressão:

$$
\mu_{e}=\operatorname{tg} \theta_{c}
$$

Assim, para a realização da atividade proposta, as expressões (2) e (4) nos permitem determinar o valor do coeficiente de atrito estático, $\mu_{\mathrm{e}}$. A expressão (2) possibilita ainda a determinação de $\mu_{e}$ por meio do coeficiente angular da reta 
obtida pelo gráfico $\operatorname{fat}_{e} X N$, o que seria o terceiro procedimento para a determinação do coeficiente de atrito estático.

\section{Material utilizado}

- Bloco de madeira $(15,0 \times 9,0 \times 2,0) \mathrm{cm}$;

- 2 réguas de madeira de $30 \mathrm{~cm}$ de comprimento;

- 2 pitões e um parafuso, pequenos;

- Placa metálica retangular de $(5,0 \times 3,0) \mathrm{cm}$;

- 5 pesos $^{2}$ de massa $100 \mathrm{~g}$ (caso se inclua mais uma determinação de $\mu$ );

- Mola com constante elástica conhecida;

- Superfície plana de madeira (tábua) de comprimento $(60,0 \times 10,0 \times 2,0) \mathrm{cm}$;

- Transferidor.

\section{Montagem}

A montagem do equipamento experimental segue os seguintes passos:

a) Inicialmente, corta-se uma das réguas de madeira em duas metades, fixando-as paralelamente sobre o bloco de madeira; em seguida, fixa-se a placa metálica sobre elas. Desse modo, a outra régua pode se mover no espaço deixado como uma gaveta e podem ser colocados pesos sobre a placa para obter outros valores de $\vec{N}$. Note que a extremidade lateral esquerda da placa pode ser usada como referência para a leitura da escala da régua que passa sob ela, como indicado na Fig. 3.

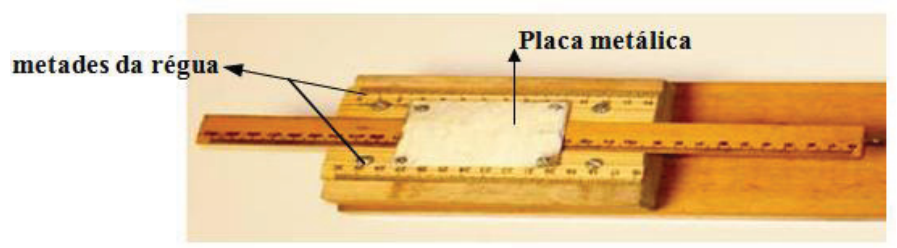

Fig. 3 - Encaixe da régua no bloco de madeira.

\footnotetext{
2 De acordo com o dicionário Houaiss eletrônico, uma das acepções da palavra peso é "pedaço de ferro ou de outro metal aferido, empregado como padrão nas balanças".
} 
b) Para completar a montagem, prende-se uma das extremidades da mola no bloco de madeira, por meio de um pitão pequeno; a outra extremidade da mola é presa abaixo da extremidade exterior da régua por meio de um parafuso pequeno. $\mathrm{Na}$ ponta da régua, prende-se um pitão para facilitar sua movimentação, como indicado na Fig. 4.

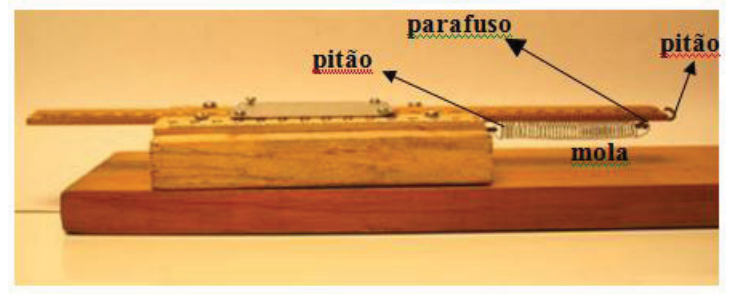

Fig. 4 - Fixação da mola no conjunto bloco-régua.

c) O conjunto bloco-régua-mola deve ser apoiado sobre a tábua.

\section{Procedimentos}

IV.1 Determinação do coeficiente de atrito estático entre duas superfícies em contato por arrasto do conjunto bloco-mola sobre uma superfície horizontal

Inicialmente, determina-se o módulo da força de atrito estático máximo. Para isso, com o conjunto bloco-régua-mola em repouso sobre a tábua colocada na horizontal, aplica-se à mola presa ao bloco de madeira uma força $\vec{F}$ de módulo variável, paralelamente à tábua, no sentido de deslocá-lo. Como indicado na Fig. 5, à medida que se alonga a mola, o módulo da força $\vec{F}$ aumenta, até que o bloco se destaque e comece a se mover.

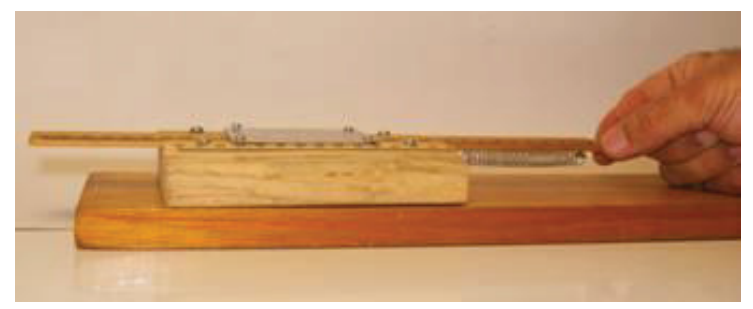

Fig. 5 - A mola é puxada até que o bloco fique na iminência do movimento. 
No momento em que o movimento do bloco é iminente, o módulo da força $\vec{F}$ é igual ao módulo da força de atrito estático máximo, $\vec{f}_{a e(m a ́ x)}$, entre as superfícies em contato do bloco e da tábua.

A medida dessa força pode ser feita em função do alongamento $\mathrm{x}$ da mola no momento em que o bloco fica na iminência de mover-se. Deve-se repetir esse procedimento algumas vezes e adotar o valor médio de x para a determinação da intensidade da força de atrito estático máximo. Como a constante elástica $k$ da mola é conhecida, determina-se o módulo dessa força por meio da Lei de Hooke $(F=k x)$.

Para a determinação do coeficiente de atrito estático a partir da expressão (2), é necessário, também, conhecer a intensidade da força normal $\vec{N}$.

Como, no caso ilustrado na Fig. 5, o módulo da força normal é igual ao módulo da força peso, podemos determiná-lo mediante dois procedimentos: medir a massa do conjunto em uma balança e depois multiplicar esse valor pelo módulo da aceleração da gravidade local ou utilizar a própria montagem e aplicar a Lei de Hooke.

Para utilizar a segunda opção, basta pendurar verticalmente o conjunto e medir o alongamento x da mola, como está indicado na Fig. 6.

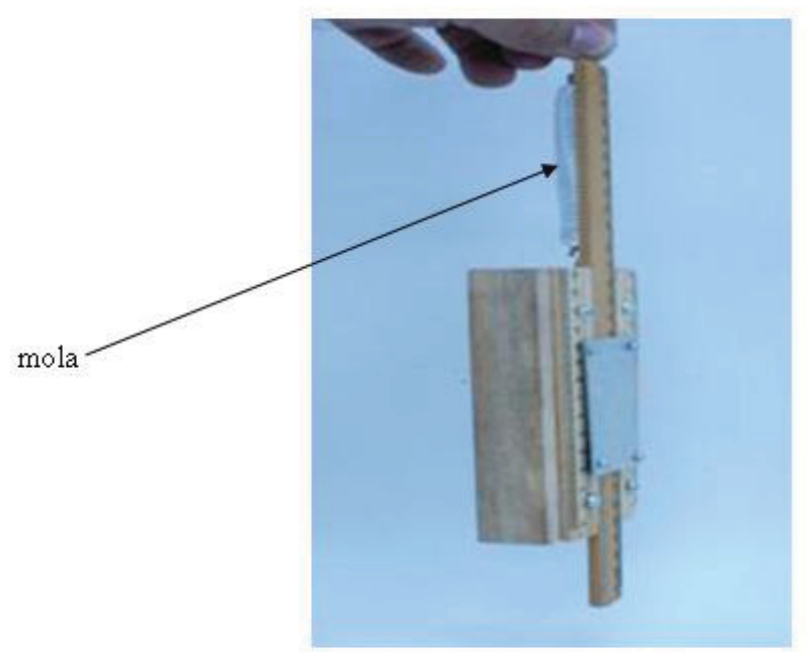

Fig. 6 - Determinação do peso do conjunto bloco-régua. 
Assim, com os módulos das forças de atrito estático máximo $f_{a e(m a ́ x)} \mathrm{e}$ normal $\mathrm{N}$, determina-se o coeficiente de atrito estático, $\mu_{e 1}$, entre o bloco e a tábua (o índice 1 indica a primeira determinação desse coeficiente), a partir da substituição de dados na expressão (2):

$$
\mu_{e 1}=\frac{f_{a e(m a ́ x)}}{N}
$$

IV.2 Determinação do coeficiente de atrito estático por meio do coeficiente angular da reta obtida do gráfico $f_{a e(m a ́ x)} X N$

Basta repetir o procedimento anterior colocando-se pesos sobre a placa metálica do bloco. Obtêm-se, assim, diferentes valores do módulo de $\vec{N}$ e dos respectivos módulos de $\vec{f}_{a e(m a ́ x)}$, que possibilitam a construção do gráfico e a determinação de $\mu_{e 2}$ (o índice 2 indica a segunda determinação desse coeficiente) por meio da reta obtida.

IV.3 Determinação do coeficiente de atrito estático de duas superfícies em contato por meio do deslizamento sobre uma superfície inclinada $\left(\mu_{e 3}\right)$

Com o conjunto bloco-régua-mola apoiado em repouso sobre a tábua, ela é inclinada paulatinamente até o momento em que ocorre a iminência do movimento, conforme está indicado na Fig. 7.

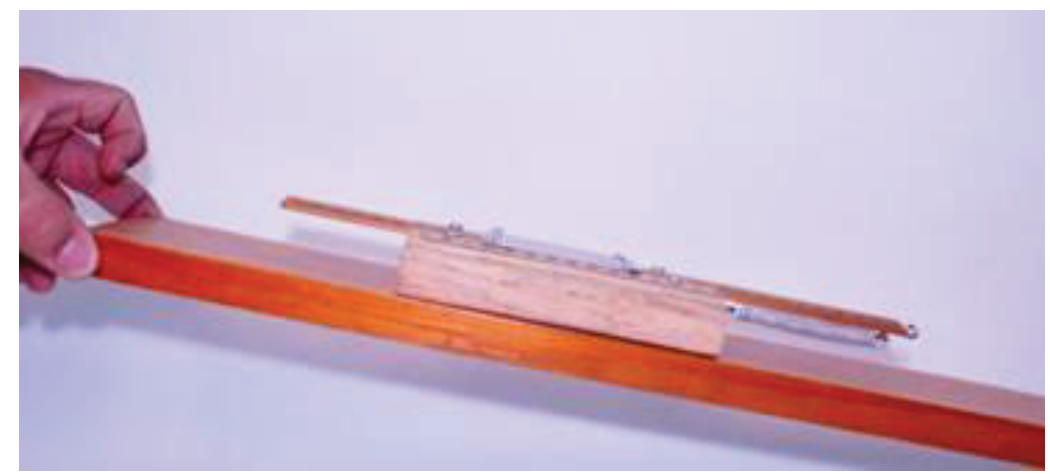

Fig. 7 - Determinação do coeficiente de atrito estático pelo ângulo crítico. 
Com auxílio do transferidor, mede-se o ângulo crítico de inclinação $\theta_{c}$ (ângulo de inclinação no momento em que o bloco fica na iminência de escorregar). Conhecido esse valor, determina-se o coeficiente de atrito estático $\mu_{e 3}$ (o índice 3 indica a terceira determinação desse coeficiente) entre o bloco e a tábua por meio da expressão (4)

$$
\mu_{e 3}=\operatorname{tg} \theta_{c}
$$

\section{Resultados obtidos}

A proposta experimental visa a determinação do coeficiente de atrito estático por três procedimentos diferentes:

- por arrasto do conjunto bloco-régua-mola sobre uma superfície horizontal $\left(\mu_{e 1}\right)$;

- mediante o coeficiente angular da reta obtida do gráfico $f_{a e(m a ́ x)} X N$ $\left(\mu_{e 2}\right) ; \mathrm{e}$

- por meio do deslizamento do conjunto bloco-régua-mola sobre uma superfície inclinada $\left(\mu_{e 3}\right)$.

V.1 Dados relativos à determinação do coeficiente de atrito estático por arrasto do conjunto bloco-mola sobre uma superfície horizontal $\left(\mu_{e 1}\right)$

$\mathrm{Na}$ atividade experimental, utilizamos uma mola cujo valor da constante elástica era igual a $1,7 \mathrm{~N} / \mathrm{m}$.

Para a determinação do valor médio da força de atrito estático máximo (Fig. 5), foram feitas quatro medidas referentes ao comprimento inicial da mola $\left(\ell_{0}\right)$ e ao comprimento final da mola $(\ell)$.

A leitura inicial do comprimento da mola, $\ell_{0}$, foi feita com ela em repouso e com o bloco sobre a tábua. Porém, a leitura do comprimento final da mola $(\ell)$ foi realizada com ela alongada até o valor no qual se percebia a iminência do movimento do bloco.

Apresentamos, na tabela 1, os dados obtidos. 
Tabela 1 - Dados relativos à determinação da força de atrito estático máximo.

\begin{tabular}{|c|c|c|c|c|}
\hline $\begin{array}{c}\mathrm{n} \\
(\text { medidas })\end{array}$ & $\begin{array}{c}\ell_{0} \\
(\mathrm{~m})\end{array}$ & $\begin{array}{c}\ell \\
(\mathrm{m})\end{array}$ & $\begin{array}{c}\mathrm{x}=\ell-\ell_{0} \\
(\mathrm{~m})\end{array}$ & $\begin{array}{c}\mathrm{F}=\mathrm{kx} \\
(\mathrm{N})\end{array}$ \\
\hline $1^{\mathrm{a}}$ & $7,0 \times 10^{-2}$ & $8,2 \times 10^{-2}$ & $1,2 \times 10^{-2}$ & $2,2 \times 10^{-2}$ \\
\hline $2^{\mathrm{a}}$ & $7,0 \times 10^{-2}$ & $8,3 \times 10^{-2}$ & $1,3 \times 10^{-2}$ & $2,3 \times 10^{-2}$ \\
\hline $3^{\mathrm{a}}$ & $7,0 \times 10^{-2}$ & $8,2 \times 10^{-2}$ & $1,2 \times 10^{-2}$ & $2,2 \times 10^{-2}$ \\
\hline $4^{\mathrm{a}}$ & $7,0 \times 10^{-2}$ & $8,3 \times 10^{-2}$ & $1,3 \times 10^{-2}$ & $2,3 \times 10^{-2}$ \\
\hline $\begin{array}{c}\text { Média das } \\
\text { medidas }\end{array}$ & $7,0 \times 10^{-2}$ & $8,3 \times 10^{-2}$ & $1,3 \times 10^{-2}$ & $2,3 \times 10^{-2}$ \\
\hline
\end{tabular}

$$
f_{\text {ae(máx) }}=F=2,3 \times 10^{-2} \mathrm{~N}
$$

Para a determinação do valor da força normal (Fig. 6), dependuramos, pela mola, o sistema bloco-régua. Aqui também realizamos quatro medidas relativas à deformação da mola, tendo em vista que o comprimento inicial da mola já havia sido determinado.

Como utilizamos a mesma mola, o valor da constante elástica $\mathrm{k}$ era igual a $1,7 \mathrm{~N} / \mathrm{m}$.

Tabela 2 - Dados relativos à determinação da força normal.

\begin{tabular}{|c|c|c|c|c|}
\hline $\begin{array}{c}\mathrm{n} \\
(\text { medidas })\end{array}$ & $\begin{array}{c}\ell_{0} \\
(\mathrm{~m})\end{array}$ & $\begin{array}{c}\ell \\
(\mathrm{m})\end{array}$ & $\begin{array}{c}\mathrm{x}=\ell-\ell_{0} \\
(\mathrm{~m})\end{array}$ & $\begin{array}{c}\mathrm{N}=\mathrm{kx} \\
(\mathrm{N})\end{array}$ \\
\hline $1^{\mathrm{a}}$ & $7,0 \times 10^{-2}$ & $11,3 \times 10^{-2}$ & $4,4 \times 10^{-2}$ & $7,5 \times 10^{-2}$ \\
\hline $2^{\mathrm{a}}$ & $7,0 \times 10^{-2}$ & $11,3 \times 10^{-2}$ & $4,3 \times 10^{-2}$ & $7,3 \times 10^{-2}$ \\
\hline $3^{\mathrm{a}}$ & $7,0 \times 10^{-2}$ & $11,4 \times 10^{-2}$ & $4,4 \times 10^{-2}$ & $7,5 \times 10^{-2}$ \\
\hline $4^{\mathrm{a}}$ & $7,0 \times 10^{-2}$ & $11,4 \times 10^{-2}$ & $4,4 \times 10^{-2}$ & $7,5 \times 10^{-2}$ \\
\hline $\begin{array}{c}\text { Média das } \\
\text { medidas }\end{array}$ & $7,0 \times 10^{-2}$ & $11,4 \times 10^{-2}$ & $4,4 \times 10^{-2}$ & $7,5 \times 10^{-2}$ \\
\hline
\end{tabular}

$$
N=7,5 \times 10^{-2} \mathrm{~N}
$$

Obtidos o módulo da força de atrito estático máximo e o módulo da força normal, utilizamos a expressão (2) para a determinação do coeficiente de atrito estático (procedimento 1).

$$
\mu_{e 1}=\frac{f_{a e(m a ́ x)}}{N}
$$




$$
\begin{aligned}
& \mu_{e 1}=\frac{2,25 \times 10^{-2}}{7,5 \times 10^{-2}} \\
& \mu_{e 1}=0,3
\end{aligned}
$$

\section{V.2 Dados relativos à determinação do coeficiente de atrito estático mediante o coeficiente angular da reta obtida do gráfico $f_{a e(m a ́ x)} X N\left(\mu_{e 2}\right)$}

A partir da montagem na situação ilustrada na Fig. 5, fomos incorporando ao conjunto pesos de massas diferentes, de forma a variar a intensidade da força normal da montagem. Consequentemente, o valor da força de atrito estático máxima foi se alterando também.

Portanto, a cada incorporação de pesos ao conjunto, era necessária uma intensidade maior de força a ser aplicada sobre a mola para que o conjunto ficasse na iminência do movimento.

Os dados relativos à variação da força normal do conjunto e ao alongamento da mola devido à força necessária para colocar o bloco na iminência do movimento são apresentados na tabela 3 a seguir.

Tabela 3 - Dados relativos à determinação do coeficiente de atrito estático pelo coeficiente angular da reta do gráfico Força de atrito estático máximo $x$ Força normal.

\begin{tabular}{|c|c|c|c|c|c|}
\hline $\begin{array}{c}\mathrm{n} \\
(\text { medidas })\end{array}$ & $\begin{array}{c}\mathrm{N} \\
(\mathrm{N})\end{array}$ & $\begin{array}{c}\ell_{0} \\
(\mathrm{~m})\end{array}$ & $\begin{array}{c}\ell \\
(\mathrm{m})\end{array}$ & $\begin{array}{c}\mathrm{x} \\
(\mathrm{m})\end{array}$ & $\begin{array}{c}\mathrm{f}_{\text {ae(máx) }}=\mathrm{kx} \\
(\mathrm{N})\end{array}$ \\
\hline $1^{\mathrm{a}}$ & $7,5 \times 10^{-2}$ & $7,0 \times 10^{-2}$ & $8,2 \times 10^{-2}$ & $1,2 \times 10^{-2}$ & $2,2 \times 10^{-2}$ \\
\hline $2^{\mathrm{a}}$ & $8,5 \times 10^{-2}$ & $7,0 \times 10^{-2}$ & $8,5 \times 10^{-2}$ & $1,5 \times 10^{-2}$ & $2,6 \times 10^{-2}$ \\
\hline $3^{\mathrm{a}}$ & $9,1 \times 10^{-2}$ & $7,0 \times 10^{-2}$ & $8,7 \times 10^{-2}$ & $1,7 \times 10^{-2}$ & $2,9 \times 10^{-2}$ \\
\hline $4^{\mathrm{a}}$ & $\begin{array}{c}10,5 \times 10^{-} \\
2\end{array}$ & $7,0 \times 10^{-2}$ & $8,9 \times 10^{-2}$ & $1,9 \times 10^{-2}$ & $3,2 \times 10^{-2}$ \\
\hline
\end{tabular}

Com base nos dados da tabela 3, podemos construir o gráfico do valor da força de atrito estático máximo pela intensidade da força normal.

Na Fig. 8, apresentamos esse gráfico, o qual foi construído utilizando o software Origin Pro 8.1.

O valor do coeficiente angular da reta média, determinado pelo software, vale 0,33 . 
V.3 Dados relativos à determinação do coeficiente de atrito estático por meio do deslizamento sobre uma superfície inclinada $\left(\mu_{e 3}\right)$

Para determinarmos o coeficiente de atrito estático a partir da determinação do ângulo crítico, colocamos o conjunto sobre a tábua, a qual, aos poucos, foi sendo inclinada (Fig. 7).

O processo de inclinação da tábua era interrompido no momento em que se percebia que o conjunto estava na iminência de escorregar.

$\mathrm{Na}$ tabela 4, apresentamos os dados relativos aos ângulos críticos para quatro medidas.

A partir desses dados, foi calculado o valor do coeficiente de atrito estático, substituindo-o na expressão (4).

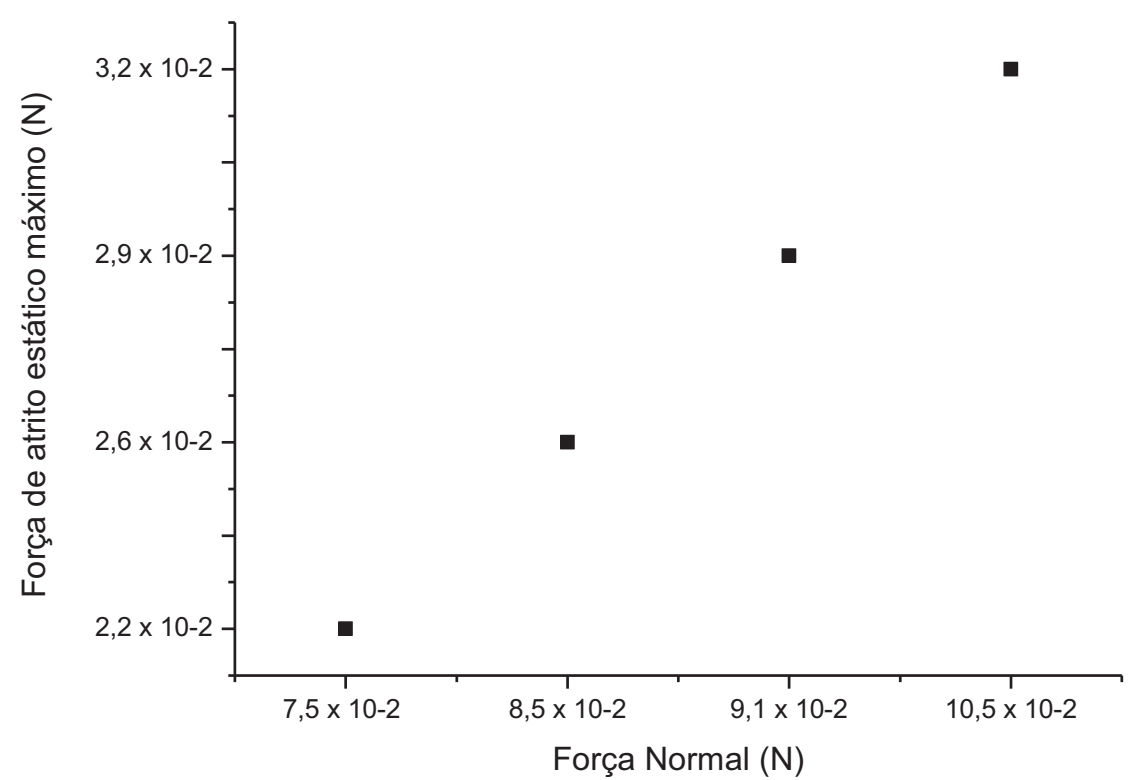

Fig. 8 - Gráfico da Força de atrito estático máximo X Força normal. 
Tabela 4 - Dados relativos à determinação do coeficiente de atrito estático pela inclinação do plano no ângulo crítico.

\begin{tabular}{|c|c|c|}
\hline $\begin{array}{c}\mathrm{n} \\
(\text { medidas })\end{array}$ & $\theta \mathrm{c}\left(^{\mathrm{o}}\right)$ & $\operatorname{tg} \theta \mathrm{c}$ \\
\hline $1^{\mathrm{a}}$ & 15 & 0,27 \\
\hline $2^{\mathrm{a}}$ & 17 & 0,31 \\
\hline $3^{\mathrm{a}}$ & 16 & 0,29 \\
\hline $4^{\mathrm{a}}$ & 17 & 0,31 \\
\hline $\begin{array}{c}\text { Média das } \\
\text { medidas }\end{array}$ & 16 & 0,29 \\
\hline
\end{tabular}

Coeficiente de atrito: $\mu_{e 3}=\operatorname{tg} \alpha=0,29$.

\section{Conclusão}

A atividade experimental proposta é de fácil construção e requer materiais simples e de baixo custo para ser montada. Como podemos observar pelos resultados obtidos, sua realização em sala de aula pode ser bastante interessante não apenas pelo fato de os resultados apresentarem uma grande coerência entre si, o que confere confiabilidade à atividade, mas também por oferecer várias oportunidades de discussão com os alunos sobre os conceitos relacionados ao fenômeno do atrito.

A proposição de cálculos referentes à diferença percentual entre os resultados obtidos pode evidenciar essa coerência e possibilitar uma discussão interessante sobre a teoria de erros e de algarismos significativos.

Em cada procedimento proposto, é possível possibilitar aos estudantes oportunidades de contextualização que, na maioria das vezes, são apenas apresentadas a eles de maneira teórica, exigindo grande abstração. Expressões como: "iminência do movimento" e "a intensidade da força de atrito estático varia de zero até um valor máximo" podem ser melhor compreendidas em cada uma das etapas propostas, pois deixam de ser apenas palavras para se constituírem em uma percepção mais concreta dessas ideias.

Além disso, a atividade permite situações nas quais os alunos podem diferenciar os conceitos relativos à força de atrito estático, força de atrito estático máximo e força de atrito dinâmico.

Apesar de não termos realizados medidas do coeficiente de atrito a partir da variação das superfícies em contato, a abordagem dessa questão pode ser proposta a partir da montagem que sugerimos. Para isso, basta que, além da tábua 
sobre o qual o conjunto bloco-régua-mola seja colocado, outras superfícies sejam utilizadas, tais como: fórmica, borracha, etc.

Da mesma forma, outras questões podem discutidas em sala de aula a partir de análises com a montagem proposta, como, por exemplo, o fato de a área de contato não interferir na intensidade da força de atrito estático máximo. Para isso, o professor pode propor ao aluno que puxe a mola, com o bloco deitado, apoiando-se lateralmente na tábua. Isso faz com que a área de contato com a tábua seja alterada. Com essa variação simples do procedimento, o aluno pode verificar que o alongamento da mola não se altera para qualquer posição do conjunto bloco-régua-mola sobre a tábua.

Outro aspecto interessante que pode ser destacado pelo professor durante a aula é que, quando o conjunto bloco-régua-mola entra em movimento, seu alongamento diminui em relação ao momento em que ele estava na iminência do movimento. Isso pode permitir uma discussão sobre o fato de a força de atrito estático máximo ter uma intensidade maior que a força de atrito dinâmico.

Finalmente, é interessante destacar que a atividade oferece muitas possibilidades de interação entre o professor, os alunos e o objeto do conhecimento.

\section{Bibliografia}

BOWDEN, F. P.; TABOR, D. The friction and lubrication of solids. London: Oxford Univ. Press, 1950.

COULOMB, C. A. Theorie des machine simples, en ayant égard au Frottement de leurs Parties, et a la Roideur des Cordages. $\mathbf{X}$ Mémories de mathématique et de physique de $\mathrm{l}^{\prime}$ Académie Royale des Sciences. Paris, p. 161-342, 1785 .

GASPAR, A. Física. Mecânica. São Paulo: Editora Ática, 2000. v. 1

MOSSMANN, V. L. F.; MELLO, K. B.; CATELLI, F.; LIBARDI, H.; DAMO, I. S. Determinação dos coeficientes de atrito estático e cinético utilizando-se a aquisição automática de dados. Revista Brasileira de Ensino de Física, v. 24, n. 2, 2002.

MATUO, C. Y.; MARINELLI, J. R. Importância do cálculo da propagação de erros em um experimento de atrito estático. Caderno Brasileiro de Ensino de Física, v. 24, n. 1, p. 132-139, 2007. 
PERSSON, B. N. J. Sliding friction: physical principles and applications. Berlin: Springer, 2000.

TOMLINSON, G. A. Molecular theory of friction. Philos. Mag., v. 7, p. $905-$ 939, 1929. 